\title{
Efficiency in Euclidean constrained location problems
}

\author{
E. Carrizosa, E. Conde, F.R. Fernandez and J. Puerto \\ Dpto. de Estadistica e Investigacion Operativa, Facultad de Matematicas, Universidad de Sevilla, Tarfia s/n, 41012 Sevilla, Spain
}

Received May 1992

Revised August 1993

In this note we present geometrical characterizations for the set of efficient, weakly efficient and properly efficient solutions to the multiobjective Euclidean Location problem with convex locational constraints, extending the known results for the unconstrained problem. It is shown that the set of the (weakly) efficient points coincides with the closest-point projection of the convex hull of the demand points onto the feasible set $S$. It is also shown that the set of properly efficient solutions is the union of two sets: the set of feasible demand points and the closest-point projection of the relative interior of the convex hull of the demand points onto $S$.

efficiency; location theory; Weber problems

\section{The model}

Let $A$ be a finite set of points in $\mathbb{R}^{n}$ (demand points). A facility is to be located at some point $x$ within a feasible set $S \subseteq \mathbb{R}^{n}$ in such a way that all the demand points have the facility as close as possible, where distances are measured by the Euclidean distance $d$ in $\mathbb{R}^{n}$ :

$$
d(x, y)=\langle x-y, x-y\rangle^{1 / 2} \text { for all } x, y \in \mathbb{R}^{n}
$$

The aim of simultaneous minimization over $S$ of the family of functions $\{d(a, \cdot): a \in A\}$ leads us to the multiobjective problem $\operatorname{MOP}(A, S)$,

$$
\operatorname{MOP}(A, S): \min _{x \in S}(d(x, a): a \in A) .
$$

A point $x \in S$ is said to be an efficient (respect. weakly efficient) solution to $\operatorname{MOP}(A, S)$ iff there exists no $y \in S$ such that

$$
d(y, a) \leq d(x, a) \forall a \in A ; d(y, a)<d(x, a) \text { for some } a \in A
$$

(respect. $d(y, a)<d(x, a)$ for all $a \in A)$.

Denote respectively by $E(A, S)$ and $\operatorname{WE}(A, S)$ the set of efficient and weakly efficient solutions to $\operatorname{MOP}(A, S)$.

Any point $x$ in $E(A, S)$ is a best-possible point, in the sense that no other point is preferred to $x$ by all the demand points. However, $E(A, S)$ may contain undesirable solutions, (see, e.g. Geoffrion, 1968) what has motivated the introduction of alternative solutionsets for $\operatorname{MOP}(A, S)$.

A popular solutionset in Location theory is the Weber set $\operatorname{PE}(A, S)$, the set of the optimal solutions to problems of the form $\min _{y \in S} \sum_{a \in A} w_{a} d(a, y)$, when $w$ varies in the set of vectors with positive components. 
By convexity of the functions $d(\cdot, a)$, as soon as the set $S$ is closed and convex, the set $\operatorname{PE}(A, S)$ coincides with the set of properly efficient points (see Geoffrion, 1968).

Throughout this note, the following notation is used: $W$ represents the set of normalized nonnegative vectors,

$$
W=\left\{\left(w_{a}\right)_{a \in A} \in \mathbb{R}^{|A|}, w_{a} \geq 0 \forall a \in A, \sum_{a \in A} w_{a}=1\right\}
$$

and $W^{+}$represent the set of normalized positive vectors,

$$
W^{+}=\left\{\left(w_{a}\right)_{a \in A} \in \mathbb{R}^{|A|}, w_{a}>0 \forall a \in A, \sum_{a \in A} w_{a}=1\right\} .
$$

For any set $X$ in $\mathbb{R}^{n}, H(X)$ represents its convex hull.

Given a nonempty closed convex set $X \subset \mathbb{R}^{n}$ and $y \in \mathbb{R}^{n}$, denote by $\operatorname{proj}_{X}(y)$ the point in $X$ closest to $y$, i.e.: $\operatorname{proj}_{X}(y)$ is the optimal solution to the optimization problem

$$
\min _{x \in X} d(x, y) \text {. }
$$

Observe that, as soon as $X$ is a nonempty closed and convex set, $\operatorname{proj}_{X}(\cdot)$ is well-defined.

For any set $Y \subseteq \mathbb{R}^{n}$, denote by $\operatorname{proj}_{X}(Y)$ the set

$$
\operatorname{proj}_{X}(Y)=\bigcup_{y \in Y} \operatorname{proj}_{X}(y) \text {. }
$$

\section{Efficient points}

Lemma 1. For any $x, y \in \mathbb{R}^{n}$, and any $w=\left(w_{a}\right)_{a \in A} \in W$, the following statements are equivalent:

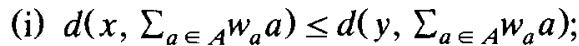

(ii) $\sum_{a \in A} w_{a} d(x, a)^{2} \leq \sum_{a \in A} w_{a} d(y, a)^{2}$.

Proof. For any $z \in \mathbb{R}^{n}$, it can be seen that $\sum_{a \in A} w_{a} d(z, a)^{2}=d\left(z, \sum_{a \in A} w_{a} a\right)^{2}-d\left(0, \sum_{a \in A} w_{a} a\right)^{2}+$ $\sum_{a \in A} w_{a} d(0, a)^{2}$. Hence

$$
\sum_{a \in A} w_{a} d(x, a)^{2} \leq \sum_{a \in A} w_{a} d(y, a)^{2}
$$

iff

$$
d\left(x, \sum_{a \in A} w_{a} a\right)^{2} \leq d\left(y, \sum_{a \in A} w_{a} a\right)^{2} \text {, i.e.: } d\left(x, \sum_{a \in A} w_{a} a\right) \leq d\left(y, \sum_{a \in A} w_{a} a\right)
$$

Theorem 1. Let $X$ be a nonempty closed convex set in $\mathbb{R}^{n}$, and let $x \in \mathbb{R}^{n}$. The following statements are equivalent:

(i) There exists no $y \in X$ such that

$$
d(y, a)<d(x, a) \text { for all } a \in A .
$$

(ii) There exists $a^{*} \in H(A)$ such that

$$
d\left(a^{*}, y\right) \geq d\left(a^{*}, a\right) \quad \text { for all } y \in X .
$$

Proof. Indeed, condition (i) is verified iff the set $Y$,

$$
Y=\left\{y \in X: d(y, a)^{2}<d(x, a)^{2} \text { for all } a \in A\right\}
$$


is empty. By Theorem 4.2.3 of Mangasarian (1969), (recall that $d(\cdot, a)$ is convex for all $a \in A$ ), $Y$ is empty iff

$$
\exists w \in W / \sum_{a \in A} w_{a} d(x, a)^{2} \leq \min _{y \in X} \sum_{a \in A} w_{a} d(y, a)^{2}
$$

which, by Lemma 1 , is equivalent to

$$
\exists w \in W / d\left(x, \sum_{a \in A} w_{a} a\right) \leq \min _{y \in X} d\left(y, \sum_{a \in A} w_{a} a\right)
$$

i.e.:

$$
\exists a^{*}\left(a^{*}=\sum_{a \in A} w_{a} a\right) \in H(A) / d\left(x, a^{*}\right) \leq \min _{y \in X} d\left(y, a^{*}\right)
$$

which is condition (ii).

Hence, (i) and (ii) are equivalent

The theorem of alternative above provides a simple characterization of the set of efficient points $E(A, S)$ as soon as $S$ is a closed and convex set in $\mathbb{R}^{n}$, extending to constrained problems the result $E\left(A, \mathbb{R}^{n}\right)=H(A)($ Kuhn, 1967).

Theorem 2. For any nonempty closed convex set $S$ in $\mathbb{R}^{n}$,

$$
W E(A, S)=E(A, S)=\operatorname{proj}_{S} H(A)
$$

Proof. As the Euclidean norm is a round norm (Thisse, Ward, Wendell, 1984), it follows that $\mathrm{WE}(A, S)=E(A, S)$.

Let $x \in S$; by definition of weak efficiency, $x \in \operatorname{WE}(A, S)$ iff there exists no $y \in S$ such that $d(y, a)<d(x, a)$ for all $a \in A$.

By Theorem 1, this condition is equivalent to

$$
\exists a^{*} \in H(A) / d\left(a^{*}, y\right) \geq d\left(a^{*}, x\right) \text { for all } y \in S
$$

i.e. (recall that $x \in S$ ):

$$
\exists a^{*} \in H(A) / x=\operatorname{proj}_{S}\left(a^{*}\right),
$$

i.e. $x \in \operatorname{proj}_{S}(H(A))$, as asserted.

\section{Properly efficient points}

Our next theorem represents the set $\operatorname{PE}(A, S)$ in terms of ri $H(A)$, the relative interior of $H(A)$.

Theorem 3. For any nonempty closed convex set $S$ in $\mathbb{R}^{n}$,

$$
P E(A, S)=(A \cap S) \bigcup \operatorname{proj}_{S} \text { ri } H(A)
$$

Proof. For any $w \in W$, consider the optimization problems $\boldsymbol{P}_{1}(w)$ and $\boldsymbol{P}_{2}(w)$,

$$
\begin{array}{ll}
\boldsymbol{P}_{1}(w): & \min _{y \in S} \sum_{a \in A} w_{a} d(y, a), \\
\boldsymbol{P}_{2}(w): & \min _{y \in S} d\left(y, \sum_{a \in A} w_{a} a\right) .
\end{array}
$$


For any $x \in S \backslash A$, let $\phi_{x}: W^{+} \rightarrow W^{+}$be the function that associates to each $w=\left(w_{a}\right)_{a \in A}$ the vector $\phi_{x}(w)$, with

$$
\left(\phi_{x}(w)\right)_{a}=\left[w_{a} / d(x, a)\right] /\left[\sum_{b \in A}\left(w_{b} / d(x, b)\right)\right]
$$

which is easily seen to be a bijection.

We first show that $x \in S \backslash A$ is an optimal solution to problem $\boldsymbol{P}_{1}(w)$ iff $x$ is an optimal solution to $\boldsymbol{P}_{2}\left(\phi_{x}(w)\right)$.

Let $x \in S \backslash A$, and let $f$ and $g$ be the functions defined as

$$
f(y)=\sum_{a \in A} w_{a} d(y, a), g(y)=\sum_{a \in A}\left(\phi_{x}(w)\right)_{a} d(y, a)^{2}
$$

As $x \notin A$, both $f$ and $g$ are convex and differentiable at $x$. Furthermore, it is readily seen that

$$
\nabla f(x)=\nabla g(x) \cdot \sum_{a \in A}\left(w_{a} / 2 d(x, a)\right)
$$

Hence, for any direction $d$, the directional derivatives of $f$ and $g$ in the direction $d$ have the same sign, what implies that $x$ is an optimal solution to $\boldsymbol{P}_{1}(w)$ iff

$$
g(y) \geq g(x) \text { for all } y \in S,
$$

what, by Lemma 1, occurs if and only if $x$ solves $\boldsymbol{P}_{2}\left(\phi_{x}(w)\right)$.

Hence, we have:

$$
x \in S \backslash A \text { solves } \boldsymbol{P}_{1}(w) \text { iff } x \text { solves } \boldsymbol{P}_{2}\left(\phi_{x}(w)\right) .
$$

We show now that $\operatorname{PE}(A, S) \subseteq \operatorname{proj}_{S}(\operatorname{ri} H(A)) \cup(A \cap S)$.

For this purpose, let $x$ be an arbitrary element of $\operatorname{PE}(A, S)$.

If $x \in A$, there is nothing to show, so we only have to consider the case $x \notin A$.

As $x \in \operatorname{PE}(A, S)$, there exists $w \in W^{+}$such that $x$ is an optimal solution to $\boldsymbol{P}_{1}(w)$. As $x \notin A,(*)$ applies, thus there exists $v\left(v=\phi_{x}(w)\right) \in W^{+}$such that $x$ is an optimal solution to $\boldsymbol{P}_{2}(v)$, i.e.:

$$
x=\operatorname{proj}_{S}\left(a^{*}\right), \text { with } a^{*}=\sum_{a \in A} v_{a} a .
$$

As $A$ is finite, one has (see, e.g. Brondsted, 1983),

$$
\text { ri } H(A)=\left\{z \in \mathbb{R}^{n} / z=\sum_{a \in A} \lambda_{a} a \text { for some } \lambda \in W^{+}\right\} \text {. }
$$

Hence, $a^{*} \in$ ri $H(A)$, thus

$$
x=\operatorname{proj}_{S}\left(a^{*}\right) \in \operatorname{proj}_{S}(\operatorname{ri} H(A)) .
$$

As $x$ was an arbitrary point in $\operatorname{PE}(A, S)$, we have

$$
\operatorname{PE}(A, S) \subseteq \operatorname{proj}_{S}(\text { ri } H(A)) \cup(A \cap S) .
$$

To show the converse, observe first that the majority theorem of Witzgall (1964) implies that $(A \cap S) \subset \operatorname{PE}(A, S)$.

On the other hand, for any $x \in \operatorname{proj}_{S}($ ri $H(A)) \backslash A,(* *)$ implies that

$$
\exists v \in W^{+} \text {such that } x \text { solves } \boldsymbol{P}_{2}(v) \text {. }
$$

As $x \notin A$, by $(*), x$ solves $\boldsymbol{P}_{1}\left(\phi_{x}(v)^{-1}\right)$, thus $x \in \operatorname{PE}(A, S)$. Hence,

$$
\operatorname{PE}(S) \supseteq \operatorname{proj}_{S}(\operatorname{ri} H(A)) \cup(A \cap S),
$$

and this completes the proof. 


\section{Extensions}

The results obtained in this paper can be extended in an straightforward manner to ellipsoidal metrics, i.e.: metrics induced by a scalar product. Indeed, for any ellipsoidal metric $d^{*}$, there exists a regular matrix $T$ such that

$$
d^{*}(x, y)=d(T x, T y) \text { for all } x, y .
$$

If we denote by $E\left(A, S ; d^{*}\right)$ (respectively $\operatorname{WE}\left(A, S ; d^{*}\right), \operatorname{PE}\left(A, S ; d^{*}\right)$ ) the set of efficient (respectively weakly and properly efficient) points to the multiobjective problem with $S$ as feasible set, $A$ as set of demand points, and distances measured by $d^{*}$, one has:

$$
\begin{aligned}
& E\left(A, S ; d^{*}\right)=T^{-1} \cdot E(T \cdot A, T \cdot S ; d), \\
& \mathrm{WE}\left(A, S ; d^{*}\right)=T^{-1} \cdot \operatorname{WE}(T \cdot A, T \cdot S ; d), \\
& \operatorname{PE}\left(A, S ; d^{*}\right)=T^{-1} \cdot \operatorname{PE}(T \cdot A, T \cdot S ; d) .
\end{aligned}
$$

On the other hand, denoting by $\operatorname{proj}_{\mathrm{S}, \mathrm{d}^{*}}$ the closest-point projection with metric $d^{*}$, one has

$$
\operatorname{proj}_{S, d^{*}}(x)=T^{-1} \cdot \operatorname{proj}_{T \cdot S, d}(T \cdot x)
$$

Hence,

$$
\mathrm{WE}\left(A, S ; d^{*}\right)=E\left(A, S ; d^{*}\right)=\operatorname{proj}_{S, d^{*}}(H(A))
$$

and

$$
\operatorname{PE}\left(A, S ; d^{*}\right)=(A \cap S) \cup \operatorname{proj}_{S, d^{*}}(\operatorname{ri}(H(A))) \text {. }
$$

Extensions of the results to nonellipsoidal metrics (e.g., $l_{p}$-metrics, with $p \neq 2$ ) are not trivial, and are now under study.

\section{References}

A. Brondsted (1983), An introduction to Convex Polytopes. Springer-Verlag, New York.

A.M. Geoffrion (1968), "Proper efficiency and the theory of vector maximization", J. Math. Anal. Appl. 22, 618-630.

H.W. Kuhn (1967), “On a pair of dual nonlinear programs", in; J. Abadie (ed.), Nonlinear programming, Wiley, New York.

O.L. Mangasarian (1969), Nonlinear Programming, McGraw-Hill, New York.

J.F. Thisse, J.E. Ward and R.E. Wendell (1984), "Some properties of Location problems with block and round norms", Oper. Res. 32, $1309-1327$.

C. Witzgall (1964), "Optimal location of a central facility, mathematical models and concepts", National Bureau of Standards Report 8388. 